\title{
First report and multilocus genotyping of Enterocytozoon bieneusi from Tibetan pigs in southwestern China
}

\author{
Run Luo ${ }^{1, \mathrm{a}}$, Leiqiong Xiang ${ }^{1, \mathrm{a}}$, Haifeng Liu ${ }^{1, \mathrm{a}}$, Zhijun Zhong ${ }^{1, \mathrm{a}}$, Li Liu ${ }^{2}$, Lei Deng ${ }^{1}$, Ling Liu ${ }^{1}$, Xiangming Huang ${ }^{2}$, \\ Ziyao Zhou ${ }^{1}$, Hualin Fu ${ }^{1}$, Yan Luo ${ }^{1}$, and Guangneng Peng ${ }^{1, *}$ \\ ${ }^{1}$ The Key Laboratory of Animal Disease and Human Health of Sichuan Province, College of Veterinary Medicine, Sichuan Agricultural \\ University, Chengdu, Sichuan Province 611130, PR China \\ 2 Chengdu Giant Panda Breeding Research Base, Chengdu, Sichuan Province 625001, PR China
}

Received 6 November 2018, Accepted 25 March 2019, Published online May 12019

\begin{abstract}
Enterocytozoon bieneusi is a common intestinal pathogen in a variety of animals. While E. bieneusi genotypes have become better-known, there are few reports on its prevalence in the Tibetan pig. This study investigated the prevalence, genetic diversity, and zoonotic potential of E. bieneusi in the Tibetan pig in southwestern China. Tibetan pig feces (266 samples) were collected from three sites in the southwest of China. Feces were subjected to PCR amplification of the internal transcribed spacer (ITS) region. Enterocytozoon bieneusi was detected in 83 (31.2\%) of Tibetan pigs from the three different sites, with 25.4\% in Kangding, 56\% in Yaan, and 26.7\% in Qionglai. Prevalence varies according to age group, from $24.4 \%$ (age $0-1$ years) to $44.4 \%$ (age 1-2 years). Four genotypes of E. bieneusi were identified: two known genotypes EbpC $(n=58)$, Henan-IV $(n=24)$ and two novel genotypes, SCT01 and SCT02 (one of each). We compare our results with a compilation of published results on the host range and geographical distribution of E. bieneusi genotypes in China. Phylogenetic analysis showed these four genotypes clustered to group 1 with zoonotic potential. Multilocus sequence typing (MLST) analysis of three microsatellites (MS1, MS3, MS7) and one minisatellite (MS4) was successful in 47, 48, 23 and 47 positive specimens and identified 10, 10, 5 and 5 genotypes at four loci, respectively. This study indicates the potential danger of E. bieneusi to Tibetan pigs in southwestern China, and offers basic advice for preventing and controlling infections.
\end{abstract}

Key words: Enterocytozoon bieneusi, Tibetan pigs, ITS gene, Multilocus genotype.

\begin{abstract}
Résumé - Premier signalement et génotypage multilocus d'Enterocytozoon bieneusi chez les porcs tibétains du sud-ouest de la Chine. Enterocytozoon bieneusi est un agent pathogène intestinal commun chez de nombreux animaux. Bien que les génotypes d'E. bieneusi soient mieux connus, il existe peu de rapports sur sa prévalence chez le porc tibétain. Cette étude a examiné la prévalence, la diversité génétique et le potentiel zoonotique d'E. bieneusi chez le porc tibétain du sud-ouest de la Chine. Des matières fécales de porcs tibétains (266 échantillons) ont été collectées sur trois sites dans le sud-ouest de la Chine. Les matières fécales ont été soumises à une amplification PCR de la région de l'espaceur interne transcrit (ITS). Enterocytozoon bieneusi a été détecté chez $83(31,2 \%)$ des porcs tibétains des trois sites différents, dont 25,4\% à Kangding, $56 \%$ à Yaan et $26,7 \%$ à Qionglai. La prévalence varie selon le groupe d'âge, de 24,4 \% (0 à 1 an) à 44,4 \% (1 à 2 ans). Quatre génotypes d'E. bieneusi ont été identifiés : deux génotypes connus, EbpC $(n=58)$, Henan-IV $(n=24)$ et deux nouveaux génotypes, SCT01 et SCT02 (un cas de chaque). Nous comparons nos résultats avec une compilation des résultats publiés sur les hôtes et la répartition géographique des génotypes d'E. bieneusi en Chine. L'analyse phylogénétique a montré que ces quatre génotypes étaient regroupés dans le groupe 1, avec potentiel zoonotique. L'analyse par typage de séquence multilocus (MLST) de trois microsatellites (MS1, MS3, MS7) et d'un minisatellite (MS4) a été réussie chez 47, 48, 23 et 47 spécimens positifs et a identifié 10, 10, 5 et 5 génotypes à quatre loci, respectivement. Cette étude indique le danger potentiel d'E. bieneusi pour les porcs tibétains du sud-ouest de la Chine et propose des conseils de base pour la prévention et le contrôle des infections.
\end{abstract}

\footnotetext{
*Corresponding author: pgn. sicau@163.com
}

${ }^{\text {a }}$ These authors contributed equally to this work. 


\section{Introduction}

Microsporidia are obligate intracellular eukaryotic pathogens, classified as fungi, which are composed of approximately 1300 species in 160 genera [7]. To date, 17 microsporidia species are known to infect humans, and of these, Enterocytozoon bieneusi is the most prevalent, accounting for over $90 \%$ of cases of human microsporidiosis [6]. Since its first detection in an HIV/AIDS patient in 1985, a growing body of literature attests to E. bieneusi expanding its range of hosts [47, 51, 52]. Infection of healthy individuals with E. bieneusi results in self-limiting diarrhea and malabsorption. However, this pathogen can cause life-threatening diarrhea in immunocompromized individuals, such as AIDS patients and transplant recipients [35]. Normally, fecal-oral routes serve as the main infection pathways in humans and animals, while human inhalation of E. bieneusi spores has also been documented [54, 58]. PCR-based molecular techniques may be used to analyze the E. bieneusi genome, and for diagnosis. Based on the nested PCR amplification of internal transcribed spacers (ITS) of small subunits of ribosomal rRNA (SSU rRNA), over 240 E. bieneusi genotypes have been identified globally $[5,56,59]$. Phylogenetic analysis reveals that these genotypes clustered into nine groups. Group 1 is considered zoonotic, and is composed of genotypes from humans and a few animals, while groups 2-9 have particular host associations or are found in wastewater [5, 51]. To better comprehend E. bieneusi genetic diversity and molecular characteristics, high-resolution multi-locus sequence typing (MLST) using three microsatellites (MS1, MS3 and MS7) and one minisatellite (MS4) as markers was used to explore genotype taxonomy and transmission routes $[9,55,56]$.

In the southwest of China, Tibetan pigs are widely kept for livelihood and are economically important for farmers, especially on the plateau. Tibetan pigs have firm black hair which differs from that of the common pig, and they are sturdy, outdoor foragers. They may act as reservoirs for E. bieneusi spores and zoonotic transmission of disease. Although much research has been carried out on E. bieneusi [10, 28, 30], few studies have examined its epidemiology or Tibetan pig-associated genomes in China [20, 57]. Tibetan pigs in southwestern China have been entirely unstudied. Therefore, this study aimed to establish the incidence and molecular characteristics of E. bieneusi in Tibetan pigs, to use ITS and MLST to evaluate its genetic diversity, and to assess the potential for zoonotic transmission of microsporidiosis between Tibetan pigs and humans.

\section{Materials and methods}

\section{Ethics statement}

The study was conducted in accordance with the Research Ethics Committee and the Animal Ethics Committee of Sichuan Agricultural University. Prior to fecal specimen collection, permission was obtained from the keepers of the animals whenever possible.

\section{Collection of Tibetan pig fecal specimens}

Fresh fecal specimens were collected from 266 Tibetan pigs during June-October 2017. Samples were obtained mainly from three cities in Sichuan province, southwestern China, including Yaan $(n=50)\left(29^{\circ} 98^{\prime} \mathrm{S}, 103{ }^{\circ} \mathrm{E}\right)$, Kangding $(n=$ 201) $\left(30^{\circ} 05^{\prime} \mathrm{S}, 101^{\circ} 4^{\prime} \mathrm{E}\right)$, and Qionglai $(n=15)\left(30^{\circ} 42^{\prime} \mathrm{S}\right.$, $103^{\circ} 47^{\prime} \mathrm{E}$ ) (Table 1). Kangding is located in a subtemperate plateau humid climate zone; Yaan and Qionglai have a subtropical humid monsoon climate and these special environments are beneficial to rear Tibetan pigs. Three farms applied intensive feeding conditions and had excellent hygiene conditions. The breeding density of Tibetan pigs in Kangding was higher than in other cities. From each farm, samples were randomly collected from at least $15 \%$ of the animals. The ages of Tibetan pigs sampled ranged from 1 to 2 years. Each specimen (approximately $200 \mathrm{mg}$ ) was collected using sterile disposable latex gloves immediately after being defecated onto the ground, and transferred into $50 \mathrm{~mL}$ plastic containers. Meanwhile, the age, gender, geographic origin, number and date of each sample was also recorded. No experimental Tibetan pigs showed diarrheic or gastrointestinal conditions. Samples were stored at $4{ }^{\circ} \mathrm{C}$ in $2.5 \%(\mathrm{w} / \mathrm{v})$ potassium dichromate.

\section{DNA extraction}

Before conducting DNA extraction, potassium dichromate was removed from the fecal samples with distilled water by centrifugation for $10 \mathrm{~min}$ at $1500 \times g$, three times. Genomic DNA was extracted from $200 \mathrm{mg}$ of washed fecal matter using the EZNA1 Stool DNA kit (Omega Biotek, Norcross, GA, USA). Prior to use in PCR analysis, DNA was stored and frozen at $-20{ }^{\circ} \mathrm{C}$.

\section{PCR amplification}

Enterocytozoon bieneusi genotypes were determined using a nested PCR amplification of the entire ITS region, and positive specimens were further detected by MLST analyses using the MS1, MS3, MS4, and MS7 loci. The primers and cycling parameters implemented for these reactions were as previously described $[9,37]$. Negative controls were included in all PCR analyses. The secondary PCR products were subjected to electrophoresis in a $1.5 \%$ agarose gel and visualized under UV light by staining the gel with GoldView (Solarbio, China).

\section{Nucleotide sequencing and phylogenetic analysis}

Secondary PCR amplicons of anticipated size were sequenced in both directions by Life Technologies (Guangzhou, China) with an ABI 3730DNA Analyzer (Applied Biosystems, Foster City, CA, USA) using the BigDye ${ }^{\circledR}$ Terminator v3.1 cycle sequencing kit. Sequence accuracy was confirmed by bidirectional sequencing, and new PCR secondary products were re-sequenced, if necessary. To identify the E. bieneusi genotype, the sequences generated were respectively aligned with known reference sequences using BLAST and ClustalX 1.83. Mega 7.0 was used to construct the phylogenetic tree using the neighbor-joining (NJ) method (the Kimura two parameter model) with 1000 bootstrap replicates 
Table 1. Factors associated with prevalence of Enterocytozoon bieneusi in Tibetan pigs in southwestern China.

\begin{tabular}{lccccc}
\hline Factor & Category & No. tested & No. positive & $(\%)(95 \%$ CI $)$ & $p$-Value \\
\hline Region & Kangding & 201 & 51 & $25.37(0.193-0.314)$ & $26.67(0.013-0.520)$ \\
& Qionglai & 15 & 4 & 28 & $56.00(0.417-0.703)$ \\
Age (years) & Yaan & 50 & 61 & $33.33(0.264-0.402)$ & $<0.01$ \\
& $0-1$ & 183 & 22 & $51.51(0.278-0.552)$ & 0.318 \\
Gender & $1-2$ & 82 & 47 & $19.57(0.138-0.254)$ & 0.003 \\
& Male & 184 & 36 & $31.20(0.256-0.368)$ \\
Total & Female & 266 & 83 & \\
\hline
\end{tabular}

Table 2. Occurrence and genotypes of E. bieneusi in Tibetan pigs from different cities in southwest China.

\begin{tabular}{|c|c|c|c|}
\hline Region & Farm ID & Prevalence $(\%)$ & Genotypes $(n)$ \\
\hline \multirow[t]{2}{*}{ Kangding } & Farm 1 & $31 / 102(30.40)$ & EbpC (18), Henan-IV $(n=13)$ \\
\hline & Farm 2 & $20 / 99(20.20)$ & EbpC (12), Henan-IV $(n=8)$ \\
\hline \multirow[t]{2}{*}{ Yaan } & Farm 3 & $14 / 28(50.00)$ & $\operatorname{EbpC}(n=14)$ \\
\hline & Farm 4 & $10 / 22(45.45)$ & $\operatorname{EbpC}(n=12), \operatorname{SCT01}(n=1), \operatorname{SCT02}(n=1)$ \\
\hline Qionglai & Farm 5 & $4 / 15(26.67)$ & $\operatorname{EbpC}(n=4)$ \\
\hline Total & & $83 / 266(31.20)$ & EbpC (58), Henan-IV $(n=23), \operatorname{SCT} 01(n=1), \operatorname{SCT} 02(n=1)$ \\
\hline
\end{tabular}

Table 3. Multilocus characterization of Enterocytozoon bieneusi isolates in Tibetan pigs in southwestern China.

\begin{tabular}{lccccccc}
\hline ITS genotype & \multicolumn{5}{c}{ Multilocus genotype } & No. of MLGs \\
\cline { 2 - 7 } & MS1 & MS3 & MS4 & MS7 & GenBank accession nos. & MLGs \\
\hline Henan-IV & Type II* & Type I* & Type III & Type II & MH142190, MH142204, MH142200, MH142212 & MLG1 \\
Henan-IV & Type I & Type III* & Type III & Type II & MH142193, MH142205, MH142200, MH142212 & MLG2 \\
Henan-IV & Type I & Type I* & Type II* & Type I* & MH142193, MH142204, MH142199, MH142210 & MLG3 \\
Henan-IV & Type II* & Type II* & Type II* & Type II & MH142195, MH142206, MH142199, MH142212 & MLG4 & 1 \\
Ebpc & Type II* & Type I* & Type II* & Type II & MH142196, MH142204, MH142199, MH142209 & MLG5 & 2 \\
Ebpc & Type X* & Type I* & Type IV* & Type I* & MH142189, MH142204, MH142203, MH142213 & MLG6 & 1 \\
Ebpc & Type I & Type I* & Type II* & Type II & MH142193, MH142204, MH142199, MH142212 & MLG7 & 1 \\
Ebpc & Type II* & Type I* & Type I & Type IV* & MH142196, MH142204, MH142201, MH142213 & MLG8 & 1 \\
Ebpc & Type III* & Type IV* & Type I & Type III* & MH142194, MH142207, MH142201, MH142209 & MLG9 & 1 \\
Ebpc & Type I & Type IV* & Type I & Type II & MH142193, MH142207, MH142201, MH142209 & MLG10 & 1 \\
\hline
\end{tabular}

* Novel genotypes.

[17]. Novel genotype(s) of E. bieneusi were named according to the established system of nomenclature [34].

\section{Statistical analysis}

The variations in E. bieneusi infection rates in Tibetan pigs between different areas, gender, and ages were compared using the Chi-square test. All tests were two-sided, with $p<0.05$ indicating statistical significance. SPSS version 22.0 was used on all data. $95 \%$ confidence intervals $(95 \% \mathrm{CIs})$ were calculated to explore the strength of the association between E. bieneusi occurrence and each factor.

\section{Nucleotide sequence accession numbers}

Representative nucleotide sequences of E. bieneusi isolates were deposited in GenBank under accession numbers from
MG581429 to MG581432 for ITS sequences and MH142189_ MH142213 for the microsatellite (MS1, MS3, and MS7) and minisatellite (MS4) loci.

\section{Results and discussion}

In the present study, of the 266 Tibetan pigs sampled, 83 were PCR-positive for E. bieneusi. Infection rates detected in Tibetan pigs were $25.4 \%, 56 \%$ and $26.6 \%$ in Kangding, Yaan and Qionglai, respectively. Differences between the three areas were significant $\left(\chi^{2}=17.648, d f=2, p<0.01\right)$ (Table 1). In addition, the male Tibetan pig groups $(17.7 \%, 47 / 266)$ had higher E. bieneusi prevalence than the female groups (13.5\%, $36 / 266)$. The difference in the infection rate was also significant $\left(\chi^{2}=8.906, d f=1, p=0.003\right)$. Although high infection rates were observed in 1-2 year-old pigs $(41.51 \%, 22 / 53)$ and $0-1$ year-olds $(33.33 \%, 61 / 183)$, these rates were not 
Table 4. Host ranges and geographical distribution of Enterocytozoon bieneusi genotypes in this study in China.

\begin{tabular}{|c|c|c|c|c|}
\hline Genotype (synonym) & Host & Location & Isolate & Reference \\
\hline \multirow[t]{49}{*}{ EbpC (E, Peru4, WL13, WL17) } & Pig & Shanghai & 3 & [9] \\
\hline & Pig & Heilongjiang & 10 & [9] \\
\hline & Pig & Heilongjiang & 3 & {$[21]$} \\
\hline & Pig & Heilongjiang & 3 & [41] \\
\hline & Pig & Jilin & 1 & [19] \\
\hline & Pig & Mongolia & 1 & [19] \\
\hline & Pig & Zhejiang & 39 & {$[45]$} \\
\hline & Pig & Guangdong & 17 & {$[45]$} \\
\hline & Pig & Yunnan & 31 & {$[45]$} \\
\hline & Tibetan pig & Sichuan & 58 & This study \\
\hline & Red panda & Shanxi & 5 & [40] \\
\hline & Human & Shanghai & 1 & [42] \\
\hline & Human & Henan & 39 & [43] \\
\hline & Human & Heilongjiang & 11 & [48] \\
\hline & Human, pig, monkey & Guangxi & 4 & [25] \\
\hline & Squirrel & Sichuan & 3 & [4] \\
\hline & Wild boar & Sichuan & 85 & [24] \\
\hline & Nonhuman primates & Hebei & 1 & {$[16]$} \\
\hline & Nonhuman primates & Hubei & 3 & [16] \\
\hline & Nonhuman primates & Hunan & 4 & [16] \\
\hline & Nonhuman primates & Being & 2 & [16] \\
\hline & Nonhuman primates & Henan & 5 & {$[15]$} \\
\hline & Water & Shanghai & 37 & [11] \\
\hline & Wastewater & Shanghai & 2 & {$[18]$} \\
\hline & Wastewater & Shanghai & 2 & [27] \\
\hline & Wastewater & Shandong & 1 & {$[18]$} \\
\hline & Wastewater & Hubei & 5 & {$[18]$} \\
\hline & Camel & Xinjiang & 23 & [33] \\
\hline & Fox & Heilongjiang & 5 & [61] \\
\hline & Mink & Hebei & 4 & [53] \\
\hline & Mink & Liaoning & 3 & [53] \\
\hline & Mink & Jilin, Heilongjiang & 6 & [3] \\
\hline & Chicken & Heilongjiang & 2 & [21] \\
\hline & Flies & Henan & 1 & [49] \\
\hline & Dog & Heilongjiang & 2 & [22] \\
\hline & Dog & Shanxi & 1 & [14] \\
\hline & Cattle & Henan, Ningxia & 6 & [23] \\
\hline & Cattle & Hubei, Tianjin & 1 & [12] \\
\hline & Goat & Yunnan & 1 & {$[2]$} \\
\hline & Calve & Xinjiang & 2 & {$[32]$} \\
\hline & Deer & Henan & 4 & {$[50]$} \\
\hline & Deer & Henan & 3 & {$[13]$} \\
\hline & Deer & Jilin & 1 & [13] \\
\hline & Human & Henan & 1 & [43] \\
\hline & Human & Heilongjiang & 3 & [48] \\
\hline & Chicken & Jilin & 2 & [21] \\
\hline & Camel & Xinjiang & 1 & [33] \\
\hline & Horse & Xinjiang & 21 & [31] \\
\hline & Cattle & Xinjiang & 2 & [32] \\
\hline \multirow[t]{7}{*}{ Henan-IV } & Nonhuman primates & Hebei & 2 & [16] \\
\hline & Nonhuman primates & Shanxi & 1 & [16] \\
\hline & Nonhuman primates & Shanghai & 1 & [16] \\
\hline & Pig & Heilongjiang & 5 & [41] \\
\hline & Pig & Shanxi & 3 & [44] \\
\hline & Pig & Yunnan & 6 & [45] \\
\hline & Tibetan pig & Sichuan & 23 & This study \\
\hline SCT01 & Tibetan pig & Sichuan & 1 & This study \\
\hline SCT02 & Tibetan pig & Sichuan & 1 & This study \\
\hline
\end{tabular}




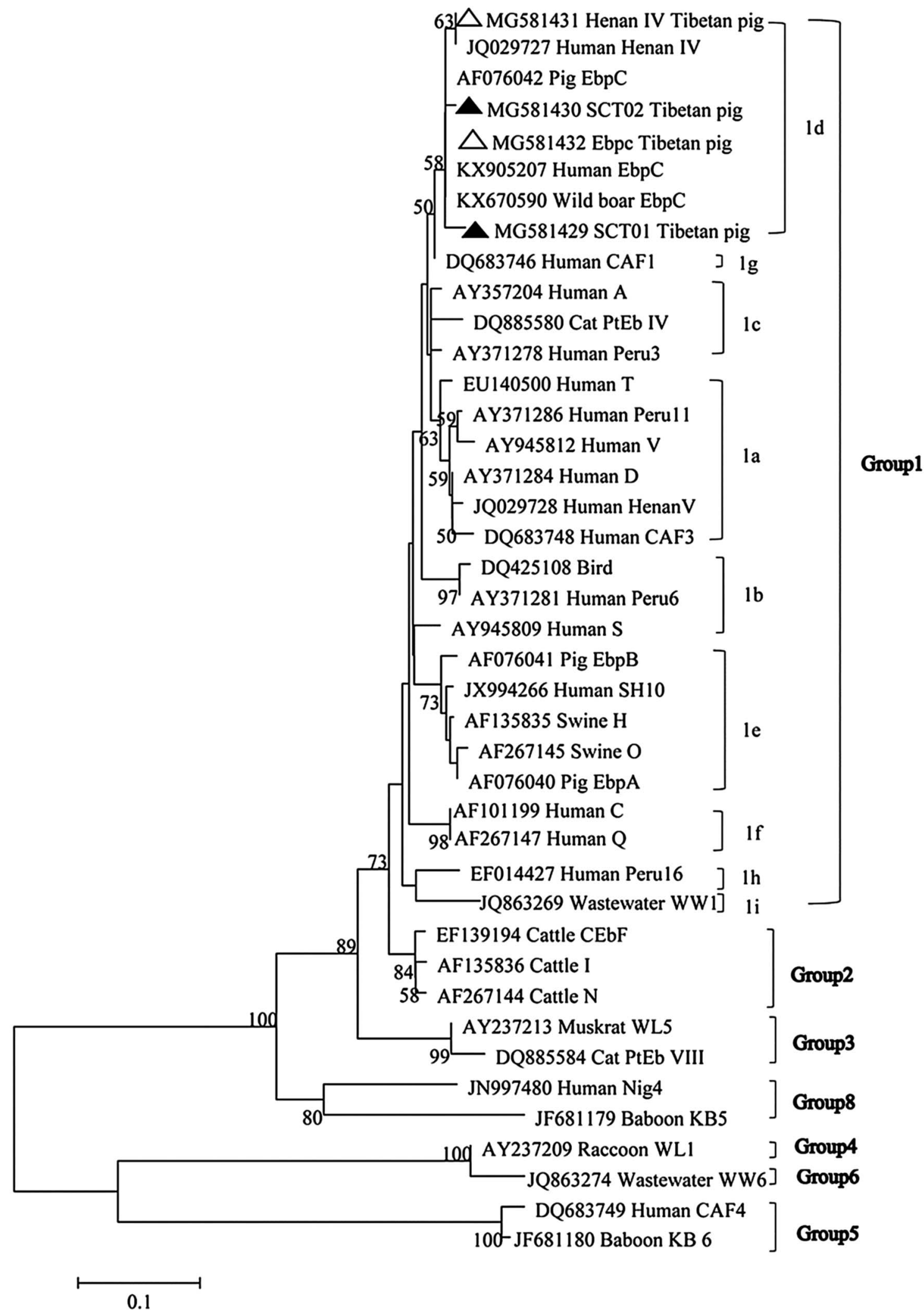

Figure 1. Phylogenetic relationship of Enterocytozoon bieneusi groups. The relationships between E. bieneusi genotypes identified in this study and other known genotypes deposited in the GenBank were inferred by a neighbor-joining analysis of ITS sequences based on genetic distance by the Kimura-2-parameter model. The numbers on the branches represent percent bootstrapping values from 1000 replicates (only bootstrap values $>50 \%$ are shown). Each sequence is identified by its accession number, genotype designation, and host origin. Genotypes with black triangles and open triangle are novel and known genotypes identified in this study, respectively. 
significantly different $\left(\chi^{2}=1.240, d f=1, p>0.05\right)$. The results of the present paper were previously published as a preprint [26]. With an overall infection rate of $31.2 \%$, this rate is lower than the documented prevalence of $E$. bieneusi for wild boars in Sichuan province, China $(41.2 \%)$, pigs in Henan province, China (45.5\%), wild boars in central Europe (33.3\%), and pigs in the State of Rio de Janeiro, Brazil (59.3\%) [10, 24, 28, 46]. However, infection rates recorded in this study were higher than those for pigs in Guangdong province, China (26.39\%), central Thailand (28.1\%), and Japan (30\%) [1, 30, 60]. Differences in infection rates between these studies may be largely attributable to climate and farming modes. Prevalence also varied across sample sites. Kangding, the only site on the Western Sichuan Plateau, had a prevalence of $25.4 \%$, possibly reflecting the area's high temperatures, and UV radiation, which may limit survival of E. bieneusi spores and reduce transmission. Other factors influencing infection levels may include geo-ecological conditions, feeding/herd densities, herd management, sample size, and the condition of host animals. Differences in prevalence in Tibetan pigs between Yaan and Kangding are thought to reflect differences between traditional and modern herd management and breeding technologies.

Nucleotide sequences from ITS-PCR were obtained from the 83 E. bieneusi-positive specimens. The epidemiology and genotypes of E. bieneusi in different areas are given in Table 2. Four genotypes were detected, including two known genotypes (EbpC, Henan-IV) and two novel genotypes, which were named SCT01 and SCT02. Genotype EbpC was the most prevalent $(21.8 \%, 58 / 266)$, and was detected in samples from all three cities. Genotype Henan-IV was only found in Kang ding $(8.6 \%, 23 / 266)$. The novel genotypes SCT01 $(0.3 \%$, $1 / 266)$ and $\operatorname{SCT} 02(0.3 \%, 1 / 266)$ were only found in single specimens, both of which came from Yaan, and are the first newly-detected E. bieneusi genotypes from Tibetan pigs. Of the four genotypes identified in this study, EbpC was the most prevalent $(69.9 \%, 58 / 83)$, and has been found in a number of animals, including cattle, dogs, cats, birds, non-human primates, bears, squirrels, sheep, foxes, deer, and humans [4, 8, 29, 36, 38, 39, 47, 51, 55]. EbpC is the prevalent E. bieneusi genotype associated with pig infection in China, reflecting E. bieneusi's dominance as a porcine parasite. In addition, we also detected 26 records of Henan-IV (solely in Yaan), a zoonotic genotype associated with human infections in Henan province in China, and to date only recorded from China, where it demonstrates strict host specificity [44], occurring only in pigs and humans. To the best our knowledge, the two genotypes EbpC and Henan-IV were identified for the first time in Tibetan pigs in the present study. This species may be a key reservoir host of these genotypes (Table 4).

Phylogenetic analysis based on ITS gene sequences of the four $E$. bieneusi genotypes obtained from the present study (two known and two novel genotypes) enabled classification for the genotypes as a single group (group 1), and further clustered into subgroup 1d, indicating zoonotic potential (Fig. 1). ITS gene sequence analysis revealed two novel genotypes, SCT01 $(n=1)$ and SCT02 $(n=1)$, both of which were detected in Yaan and clustered into group 1 zoonotic genotypes with public health significance. Other genotypes in this group include Henan-III in humans and EbpC from humans or wild boars [24, 43, 55]. Modes of transmission and zoonotic potential of $E$. bieneusi genotypes remain poorly known, and further molecular epidemiology studies are required. MLST holds promise for ongoing investigation of E. bieneusi taxonomy and genetic diversity [9]. Positive specimens were further characterized by PCR analyses of MS4, MS1, MS3 and MS7 to improve taxonomy and population genotypes of E. bieneusi. In all, 47, 48, 23 and 47 E. bieneusi isolates were amplified at the MS1, MS3, MS4, and MS7 loci, respectively, but only 12 samples were PCR-positive simultaneously at all four loci. Four distinct MLGs were observed in Henan-IV and six distinct MLGs in EbpC, named MLG1-4 and MLG5-10, respectively (Table 3). Nine, five, three and four novel types were detected at MS1, MS3, MS4 and MS7 loci, respectively. Analysis of 12 samples at four gene loci identified eight novel MLGs, including three genotype EbpC MLGs and five genotype Henan-IV MLGs (Table 3). These results reveal high genetic diversity in the Henan-IV and EbpC genotypes of E. bieneusi in Tibetan pigs.

\section{Conclusions}

This study revealed an average E. bieneusi infection rate of $31.2 \%$ in three cities in Sichuan province, and is the first report of EbpC and Henan-IV in Tibetan pigs in China. Genetic diversity was characterized using MLST, and ten MLGs were identified. These results identify Tibetan pigs as possible vectors for zoonotic transmission of human microsporidiosis. Tibetan pigs are bred widely and there is frequent human contact, making them a significant public health risk in southwest China. Thus, measures are needed to control the transmission of E. bieneusi and to develop effective vaccines and drugs for use in the event of widespread human microsporidiosis.

\section{Competing interests}

The authors declare that they have no competing interests.

\section{Funding}

This work was supported by the Chengdu Giant Panda Breeding Research Foundation (CPF2017-12).

\section{References}

1. Abe N, Kimata I. 2010. Molecular survey of Enterocytozoon bieneusi in a Japanese porcine population. Vector Borne \& Zoonotic Diseases, 10, 425.

2. Chen D, Wang SS, Zou Y, Li Z, Xie SC, Shi LQ, Zou FC, Zhu XQ, Yang JF, Zhao GH. 2018. Prevalence and multi-locus genotypes of Enterocytozoon bieneusi in black-boned sheep and goats in Yunnan Province, southwestern China. Infection, Genetics and Evolution, 65, 385-391, S1567134818306397.

3. Cong W, Qin SY, Meng QF. 2018. Molecular characterization and new genotypes of Enterocytozoon bieneusi in minks (Neovison vison) in China. Parasite, 25, 34.

4. Deng L, Li W, Yu X, Gong C, Liu X, Zhong Z, Xie N, Lei S, Yu J, Fu H, Chen H, Xu H, Hu Y, Peng G. 2016. First report of 
the human-pathogenic Enterocytozoon bieneusi from RedBellied Tree Squirrels (Callosciurus erythraeus) in Sichuan, China. PLoS One, 11, e0163605.

5. Deng L, Li W, Zhong Z, Gong C, Liu X, Huang X, Xiao L, Zhao R, Wang W, Feng F. 2016. Molecular characterization and multilocus genotypes of Enterocytozoon bieneusi among horses in southwestern China. Parasites \& Vectors, 9, 561.

6. Didier ES, Weiss LM. 1969. Microsporidiosis: not just in AIDS patients. Current Opinion in Infectious Diseases, 24, 490-495.

7. Didier ES, Weiss LM. 2006. Microsporidiosis: current status. Current Opinion in Infectious Diseases, 19, 485.

8. Ding S, Huang W, Qin Q, Tang J, Liu H. 2018. Genotype identification and phylogenetic analysis of Enterocytozoon bieneusi isolates from stool samples of diarrheic children. Journal of Parasitology, 104, 297.

9. Feng Y, Li N, Dearen T, Lobo ML, Matos O, Cama V, Xiao L. 2011. Development of a multilocus sequence typing tool for high-resolution genotyping of Enterocytozoon bieneusi. Applied \& Environmental Microbiology, 77, 4822-4828.

10. Fiuza VR, Oliveira FC, Fayer R, Santín M. 2015. First report of Enterocytozoon bieneusi in pigs in Brazil. Parasitology International, 64, 18-23.

11. Hu Y, Feng Y, Huang C, Xiao L. 2014. Occurrence, source, and human infection potential of Cryptosporidium and Enterocytozoon bieneusi in drinking source water in Shanghai, China, during a pig carcass disposal incident. Environmental Science \& Technology, 48, 14219-14227.

12. Hu S, Liu Z, Yan F, Zhang Z, Zhang G, Zhang L, Jian F, Zhang S, Ning C, Wang R. 2017. Zoonotic and host-adapted genotypes of Cryptosporidium spp., Giardia duodenalis and Enterocytozoon bieneusi in dairy cattle in Hebei and Tianjin, China. Veterinary Parasitology, 248, 68-73.

13. Huang J, Zhang Z, Yang Y, Wang R, Zhao J, Jian F, Ning C, Zhang L. 2017. New genotypes of Enterocytozoon bieneusi isolated from Sika Deer and Red Deer in China. Frontiers in Microbiology, 8, 879.

14. Karim MR, Dong H, Li T, Yu F, Li D, Zhang L, Li J, Wang R, $\mathrm{Li} \mathrm{S}, \mathrm{Li}$ X. 2015. Predomination and new genotypes of Enterocytozoon bieneusi in captive nonhuman primates in zoos in China: high genetic diversity and zoonotic significance. Plos One, 10, e0117991.

15. Karim MR, Dong H, Yu F, Jian F, Zhang L, Wang R, Zhang S, Rume FI, Ning C, Xiao L. 2014. Genetic diversity in Enterocytozoon bieneusi isolates from dogs and cats in China: host specificity and public health implications. Journal of Clinical Microbiology, 52, 3297-3302.

16. Karim MR, Wang R, Dong H, Zhang L, Li J, Zhang S, Rume FI, Qi M, Jian F, Sun M, Yang G, Zou F, Ning C, Xiao L. 2014. Genetic polymorphism and zoonotic potential of Enterocytozoon bieneusi from nonhuman primates in China. Applied \& Environmental Microbiology, 80, 1893.

17. Kumar S, Stecher G, Tamura K. 2016. MEGA7: molecular evolutionary genetics analysis version 7.0 for bigger datasets. Molecular Biology and Evolution, 33, 1870-1874.

18. Li N, Xiao L, Wang L, Zhao S, Zhao X, Duan L, Guo M, Liu L, Feng Y. 2012. Molecular surveillance of Cryptosporidium spp., Giardia duodenalis, and Enterocytozoon bieneusi by genotyping and subtyping parasites in wastewater. PLoS Neglected Tropical Diseases, 6, e1809.

19. Li W, Diao R, Yang Xiao L, Lu Y, Li Y, Song M. 2014. High diversity of human-pathogenic Enterocytozoon bieneusi genotypes in swine in northeast China. Parasitology Research, 113, 1147.

20. Li W, Li Y, Li W, Yang J, Song M, Diao R, Jia H, Lu Y, Zheng J, Zhang X. 2014. Genotypes of Enterocytozoon bieneusi in livestock in China: high prevalence and zoonotic potential. Plos One, 9, e97623.

21. Li W, Tao W, Jiang Y, Diao R, Yang J, Xiao L. 2014 Genotypic distribution and phylogenetic characterization of Enterocytozoon bieneusi in diarrheic chickens and pigs in multiple cities, China: potential zoonotic transmission. Plos One, 9, e108279.

22. Li W, Li Y, Song M, Lu Y, Yang J, Tao W, Jiang Y, Wan Q, Zhang S, Xiao L. 2015. Prevalence and genetic characteristics of Cryptosporidium, Enterocytozoon bieneusi and Giardia duodenalis in cats and dogs in Heilongjiang province, China. Veterinary Parasitology, 208, 125-134.

23. Li J, Luo N, Wang C, Qi M, Cao J, Cui Z, Huang J, Wang R, Zhang L. 2016. Occurrence, molecular characterization and predominant genotypes of Enterocytozoon bieneusi in dairy cattle in Henan and Ningxia, China. Parasites \& Vectors, 9, 1-5.

24. Li W, Deng L, Wu K, Huang X, Song Y, Su H, Hu Y, Fu H, Zhong Z, Peng G. 2017. Presence of zoonotic Cryptosporidium scrofarum., Giardia duodenalis assemblage A and Enterocytozoon bieneusi genotypes in captive Eurasian wild boars (Sus scrofa) in China: potential for zoonotic transmission. Parasites \& Vectors, 10, 10.

25. Liu H, Jiang Z, Yuan Z, Yin J, Wang Z, Yu B, Zhou D, Shen Y, Cao J. 2017. Infection by and genotype characteristics of Enterocytozoon bieneusi in HIV/AIDS patients from Guangxi Zhuang autonomous region, China. BMC Infectious Diseases, 17,684 .

26. Luo R, Xiang L, Liu H, Zhong Z, Liu L, Deng L, Song Y, Liu L, Huang X, Zhou Z, Fu H, Luo Y. 2018. First report and multilocus genotyping of Enterocytozoon bieneusi from Tibetan pigs in southwestern China. DOI: 10.1101/327767.

27. Ma J, Feng Y, Hu Y, Villegas EN, Xiao L. 2016. Human infective potential of Cryptosporidium spp., Giardia duodenalis and Enterocytozoon bieneusi in urban wastewater treatment plant effluents. Journal of Water \& Health, 14, 411-423.

28. Nemejc K, Sak B, Kvetonová D, Hanzal V, Janiszewski P, Forejtek P, Rajský D, Kotková M, Ravaszová P, Mcevoy J. 2014. Prevalence and diversity of Encephalitozoon spp. and Enterocytozoon bieneusi in wild boars (Sus scrofa) in Central Europe. Parasitology Research, 113, 761.

29. Piekarska J, Kicia M, Wesołowska M, Kopacz Ż, Gorczykowski M, Szczepankiewicz B, Kvac M, Sak B. 2017. Zoonotic microsporidia in dogs and cats in Poland. Veterinary Parasitology, 246, 108-111.

30. Prasertbun R, Mori H, Pintong AR, Sanyanusin S, Popruk S, Komalamisra C, Changbunjong $\mathrm{T}$, Buddhirongawatr $\mathrm{R}$, Sukthana Y, Mahittikorn A. 2016. Zoonotic potential of Enterocytozoon genotypes in humans and pigs in Thailand. Veterinary Parasitology, 233, 73-79.

31. Qi M, Jing B, Jian F, Wang R, Zhang S, Wang H, Ning C, Zhang L. 2017. Dominance of Enterocytozoon bieneusi genotype $\mathbf{J}$ in dairy calves in Xinjiang, Northwest China. Parasitology International, 66, 960-963.

32. Qi M, Li J, Zhao A, Cui Z, Wei Z, Jing B, Zhang L. 2018. Host specificity of Enterocytozoon bieneusi genotypes in Bactrian camels (Camelus bactrianus) in China. Parasites \& Vectors, 11, 219.

33. Qi M, Wang R, Wang H, Jian F, Li J, Zhao J, Dong H, Zhu H, Ning C, Zhang L. 2016. Enterocytozoon bieneusi genotypes in grazing horses in China and their zoonotic transmission potential. Journal of Eukaryotic Microbiology, 63, 591-597.

34. Santin M, Fayer R. 2010. Enterocytozoon bieneusi genotype nomenclature based on the internal transcribed spacer sequence: a consensus. Journal of Eukaryotic Microbiology, $56,34-38$. 
35. Santin M, Fayer R. 2011. Microsporidiosis: Enterocytozoon bieneusi in domesticated and wild animals. Research in Veterinary Science, 90, 363-371.

36. Shi K, Li M, Wang X, Li J, Karim MR, Wang R, Zhang L, Jian F, Ning C. 2016. Molecular survey of Enterocytozoon bieneusi in sheep and goats in China. Parasites \& Vectors, 9, 23.

37. Sulaiman IM, Ronald F, Lal AA, Trout JM, Schaefer FW, Lihua X. 2003. Molecular characterization of microsporidia indicates that wild mammals harbor host-adapted Enterocytozoon spp. as well as human-pathogenic Enterocytozoon bieneusi. Applied \& Environmental Microbiology, 69, 4495.

38. Tang C, Cai M, Wang L, Guo Y, Li N, Feng Y, Xiao L. 2018. Genetic diversity within dominant Enterocytozoon bieneusi genotypes in pre-weaned calves. Parasites \& Vectors, 11, 170.

39. Tavalla M, Mardani-Kateki M, Abdizadeh R, Soltani S, Saki J. 2017. Molecular diagnosis of potentially human pathogenic Enterocytozoon bieneusi and Encephalitozoon species in exotic birds in Southwestern Iran. Journal of Infection and Public Health, 11, 192-196.

40. Tian GR, Zhao GH, Du SZ, Hu XF, Wang HB, Zhang LX, Yu SK. 2015. First report of Enterocytozoon bieneusi from giant pandas (Ailuropoda melanoleuca) and red pandas (Ailurus fulgens) in China. Infection, Genetics and Evolution, 34, 32-35.

41. Wan Q, Lin Y, Mao Y, Yang Y, Li Q, Zhang S, Jiang Y, Tao W, Li W. 2016. High prevalence and widespread distribution of zoonotic Enterocytozoon bieneusi genotypes in swine in Northeast China: implications for public health. Journal of Eukaryotic Microbiology, 63, 162-170.

42. Wang L, Xiao L, Duan L, Ye J, Guo Y, Guo M, Liu L, Feng Y. 2013. Concurrent infections of Giardia duodenalis, Enterocytozoon bieneusi, and Clostridium difficile in children during a cryptosporidiosis outbreak in a pediatric hospital in China. PLoS Neglected Tropical Diseases, 7(9), e2437.

43. Wang L, Zhang H, Zhao X, Zhang L, Zhang G, Guo M, Liu L, Feng Y, Xiao L. 2013. Zoonotic Cryptosporidium species and Enterocytozoon bieneusi genotypes in HIV-positive patients on antiretroviral therapy. Journal of Clinical Microbiology, 51, 557-563.

44. Wang SS, Li JQ, Li YH, Wang XW, Fan XC, Liu X, Li ZJ, Song JK, Zhang LX, Zhao GH. 2018. Novel genotypes and multilocus genotypes of Enterocytozoon bieneusi in pigs in northwestern China: a public health concern. Infection, Genetics and Evolution, 63, 89-94.

45. Wang SS, Wang RJ, Fan XC, Liu TL, Zhang LX, Zhao GH. 2018. Prevalence and genotypes of Enterocytozoon bieneusi in China. Acta Tropica, 183, 142-152.

46. Wang H, Zhang Y, Wu Y, Li J, Qi M, Li T, Wang J, Wang R, Zhang S, Jian F. 2018. Occurrence, molecular characterization and assessment of zoonotic risk of Cryptosporidium spp., Giardia duodenalis, and Enterocytozoon bieneusi in pigs in Henan, Central China. Journal of Eukaryotic Microbiology, 65, 893-901.

47. Wu J, Han JQ, Shi LQ, Zou Y, Li Z, Yang JF, Huang CQ, Zou FC. 2018. Prevalence, genotypes, and risk factors of Enterocytozoon bieneusi in Asiatic black bear (Ursus thibetanus) in Yunnan Province, Southwestern China. Parasitology Research, 117, 1-7.

48. Yang J, Song M, Wan Q, Li Y, Lu Y, Jiang Y, Tao W, Li W. 2014. Enterocytozoon bieneusi genotypes in children in
Northeast China and assessment of risk of zoonotic transmission. Journal of Clinical Microbiology, 52, 4363.

49. Yu F, Qi M, Zhao Z, Lv C, Wang Y, Wang R, Zhang L. 2018. The potential role of synanthropic rodents and flies in the transmission of Enterocytozoon bieneusi on a dairy cattle farm in China. Journal of Eukaryotic Microbiology. DOI: 10.1111/jeu.12687.

50. Zhang Z, Huang J, Karim MR, Zhao J, Dong H, Ai W, Li F, Zhang L, Wang R. 2015. Zoonotic Enterocytozoon bieneusi genotypes in Pere David's deer (Elaphurus davidianus) in Henan, China. Experimental Parasitology, 155, 46-48.

51. Zhang XX, Cong W, Lou ZL, Ma JG, Zheng WB, Yao QX, Zhao Q, Zhu XQ. 2016. Prevalence, risk factors and multilocus genotyping of Enterocytozoon bieneusi in farmed foxes (Vulpes lagopus), Northern China. Parasites \& Vectors, 9, 1-7.

52. Zhang Q, Cai J, Li P, Wang L, Guo Y, Li C, Lei M, Feng Y, Xiao L. 2018. Enterocytozoon bieneusi genotypes in Tibetan sheep and yaks. Parasitology Research, 117, 1-7.

53. Zhang XX, Jiang RL, Ma JG, Xu C, Zhao Q, Hou G, Liu GH. 2018. Enterocytozoon bieneusi in Minks (Neovison vison) in Northern China: a Public Health Concern. Frontiers in Microbiology, 9, 1221.

54. Zhang Y, Koehler AV, Wang T, Haydon SR, Gasser RB. 2018. First detection and genetic characterisation of Enterocytozoon bieneusi in wild deer in Melbourne's water catchments in Australia. Parasites \& Vectors, 11, 2.

55. Zhong Z, Li W, Deng L, Song Y, Wu K, Tian Y, Huang X, $\mathrm{Hu}$ Y, Fu H, Geng Y. 2017. Multilocus genotyping of Enterocytozoon bieneusi derived from nonhuman primates in southwest China. Plos One, 12, e0176926.

56. Zhong Z, Tian Y, Song Y, Deng L, Li J, Ren Z, Ma X, Gu X, He C, Geng Y. 2017. Molecular characterization and multilocus genotypes of Enterocytozoon bieneusi from captive red kangaroos (Macropus Rfus) in Jiangsu province, China. Plos One, 12, e0183249.

57. Zhao W, Zhang W, Yang F, Cao J, Liu H, Yang D, Shen Y, Liu A. 2014. High prevalence of Enterocytozoon bieneusi in asymptomatic pigs and assessment of zoonotic risk at the genotype level. Applied \& Environmental Microbiology, 80, 3699-3707.

58. Zhao GH, Du SZ, Wang HB, Hu XF, Deng MJ, Yu SK, Zhang LX, Zhu XQ. 2015. First report of zoonotic Cryptosporidium spp., Giardia intestinalis and Enterocytozoon bieneusi in golden takins (Budorcas taxicolor bedfordi). Infection, Genetics and Evolution, 34, 394-401.

59. Zhao W, Wang J, Yang Z, Liu A. 2017. Dominance of the Enterocytozoon bieneusi genotype BEB6 in red deer (Cervus elaphus) and Siberian roe deer (Capreolus pygargus) in China and a brief literature review. Parasite, 24, 54.

60. Zou Y, Hou JL, Li FC, Zou FC, Lin RQ, Ma JG, Zhang XX, Zhu XQ. 2018. Prevalence and genotypes of Enterocytozoon bieneusi in pigs in southern China. Infection, Genetics and Evolution, 66, 52-56.

61. Zhao W, Zhang W, Yang Z, Liu A, Zhang L, Yang F, Wang R, Ling H. 2015. Correction: Genotyping of Enterocytozoon bieneusi in farmed Blue Foxes (Alopex lagopus) and Raccoon Dogs (Nyctereutes procyonoides) in China. Plos One, 10(11), $\mathrm{e} 0143992$.

Cite this article as: Luo R, Xiang L, Liu H, Zhong Z, Liu L, Deng L, Liu L, Huang X, Zhou Z, Fu H, Luo Y \& Peng G. 2019. First report and multilocus genotyping of Enterocytozoon bieneusi from Tibetan pigs in southwestern China. Parasite 26, 24. 
Reviews, articles and short notes may be submitted. Fields include, but are not limited to: general, medical and veterinary parasitology; morphology, including ultrastructure; parasite systematics, including entomology, acarology, helminthology and protistology, and molecular analyses; molecular biology and biochemistry; immunology of parasitic diseases; host-parasite relationships; ecology and life history of parasites; epidemiology; therapeutics; new diagnostic tools.

All papers in Parasite are published in English. Manuscripts should have a broad interest and must not have been published or submitted elsewhere. No limit is imposed on the length of manuscripts.

Parasite (open-access) continues Parasite (print and online editions, 1994-2012) and Annales de Parasitologie Humaine et Comparée (1923-1993) and is the official journal of the Société Française de Parasitologie. 